\title{
Experimental and Theoretical Study of the Rotation of Si Ad-dimers on the Si(100) Surface
}

\author{
Swartzentruber, B. S.; Smith, A. P.; Jonsson, Hannes
}

Published in:

Physical Review Letters

Link to article, DOI:

10.1103/PhysRevLett.77.2518

Publication date:

1996

Document Version

Publisher's PDF, also known as Version of record

Link back to DTU Orbit

Citation (APA):

Swartzentruber, B. S., Smith, A. P., \& Jonsson, H. (1996). Experimental and Theoretical Study of the Rotation of Si Ad-dimers on the Si(100) Surface. Physical Review Letters, 77(12), 2518-2521.

https://doi.org/10.1103/PhysRevLett.77.2518

\section{General rights}

Copyright and moral rights for the publications made accessible in the public portal are retained by the authors and/or other copyright owners and it is a condition of accessing publications that users recognise and abide by the legal requirements associated with these rights.

- Users may download and print one copy of any publication from the public portal for the purpose of private study or research.

- You may not further distribute the material or use it for any profit-making activity or commercial gain

- You may freely distribute the URL identifying the publication in the public portal 


\title{
Experimental and Theoretical Study of the Rotation of Si Ad-dimers on the Si(100) Surface
}

\author{
B. S. Swartzentruber, ${ }^{1}$ A. P. Smith, ${ }^{2}$ and H. Jónsson ${ }^{2,3}$ \\ ${ }^{1}$ Sandia National Laboratories, Albuquerque, New Mexico 87185-1413 \\ ${ }^{2}$ Department of Chemistry, University of Washington, Box 351700, Seattle, Washington 98195-1700 \\ ${ }^{3}$ CAMP, Department of Physics, Technical University of Denmark, DK-2800 Lyngby, Denmark
}

(Received 22 January 1996)

\begin{abstract}
Scanning tunneling microscopy measurements and first principles density functional theory calculations are used to study the rate of the rotational transition of Si ad-dimers on top of the surface dimer rows of $\mathrm{Si}(100)$. The rotation rate and the relative population of the two stable orientations of the ad-dimers are measured as a function of the applied electric field to extract the zero-field behavior. The measured relative stability of the two configurations is used to test the accuracy of various functionals for density functional theory calculations. [S0031-9007(96)01214-8]

PACS numbers: 68.10.Jy, 61.16.Ch, 71.10.-w, 73.20.-r
\end{abstract}

Studies of the dynamics of $\mathrm{Si}$ adatoms on Si surfaces are essential for understanding low temperature epitaxial Si crystal growth, where the system is far from equilibrium and the surface morphology is determined mainly by the rates of competing atomic transitions. An understanding of $\mathrm{Si}$ growth is an essential starting point for controlled growth of $\mathrm{Si}-\mathrm{Ge}$ heterostructures and other more complex systems. Surface morphologies observed in recent scanning tunneling microscopy (STM) images after low temperature deposition of a fraction of a monolayer of Si indicate that the dynamics of adatoms at the surface are quite complex. Isolated adatoms preferentially sit at nonepitaxial sites [1]. But, even at $160 \mathrm{~K}$ adatoms on top of the dimer rows of the reconstructed $\mathrm{Si}(100)$ surface are mobile [2] and nearly half the deposited atoms at a coverage of $2 \%$ are found as dimers sitting on top of the rows [1]. In the temperature regime around $500 \mathrm{~K}$, deposited atoms form epitaxial rows of dimers [3], but at $400 \mathrm{~K}$ mainly nonepitaxial strings of atoms are formed [4]. Around room temperature adatoms quickly pair up to form strongly bound ad-dimers, but these are then prevented from forming larger structures by the large barriers for ad-dimer diffusion [5] and the large dissociation energy of ad-dimers [6].

Calculations of Si surface dynamics that include surface relaxation and electron correlation and that are converged with respect to the basis set can at present only be carried out within density functional theory (DFT) [2]. However, at this time, such calculations are not reliable enough to give an unambiguous picture of the potential energy landscape. In order to model the dynamics of low temperature deposition, energy barriers for the atomic transitions need to be known with accuracy better than $0.1 \mathrm{eV}$. It is therefore essential that direct experimental measurements of surface dynamics be carried out to provide information about the kinetics and to provide a testing ground for theoretical calculations. Previously, kinetic parameters relevant to silicon crystal growth have been mainly extracted indirectly from comparison of the prediction of growth models with observed surface morphology [3]. In principle STM imaging can provide direct information about kinetic processes [7-9], but such experiments are limited by the rate at which dynamic events can be resolved and the results could be strongly influenced by the applied electric fields during STM imaging. Detailed and careful measurements of some atomic transitions on the $\mathrm{Si}(100)$ surface are needed to test various theoretical approaches. Theoretical calculations can then be used to get a more complete picture of the energy landscape and the various dynamical processes relevant to growth.

In this Letter we present a contribution towards such an effort. We perform direct measurements of the rate of rotation of Si ad-dimers, carefully analyzing the dependence on the applied field and compare the measured results with various levels of DFT calculations.

Two stable conformations for the ad-dimer on top of a dimer row were predicted by Brocks, Kelly, and Car [10] who carried out DFT calculations of various dimer configurations using the local density approximation (LDA). The two configurations are shown in Fig. 1. They found a preference for the $U-U$ configuration, where the ad-dimer is oriented parallel to the underlying dimers. The $P-P$ configuration, which has the ad-dimer oriented perpendicular to the underlying dimers, more consistent with epitaxial growth, was found to be $0.1 \mathrm{eV}$ higher in energy [11]. (The nomenclature here is derived from the designation of corresponding adatom sites introduced in Ref. [2].) Zhang et al. [12] carried out calculations using the empirical Stillinger-Weber (SW) potential function [13] and found an energy preference for the $P-P$ configuration by $0.05 \mathrm{eV}$. The presence of the two stable configurations was confirmed in STM images in two laboratories and it was found that the rotational transition occurs on the time scale of seconds at room temperature $[4,12]$. From the relative occupations of the two configurations at room temperature the $P-P$ configuration was estimated to be $0.06 \pm 0.01 \mathrm{eV}[4,12]$ more stable than the $U-U$ 


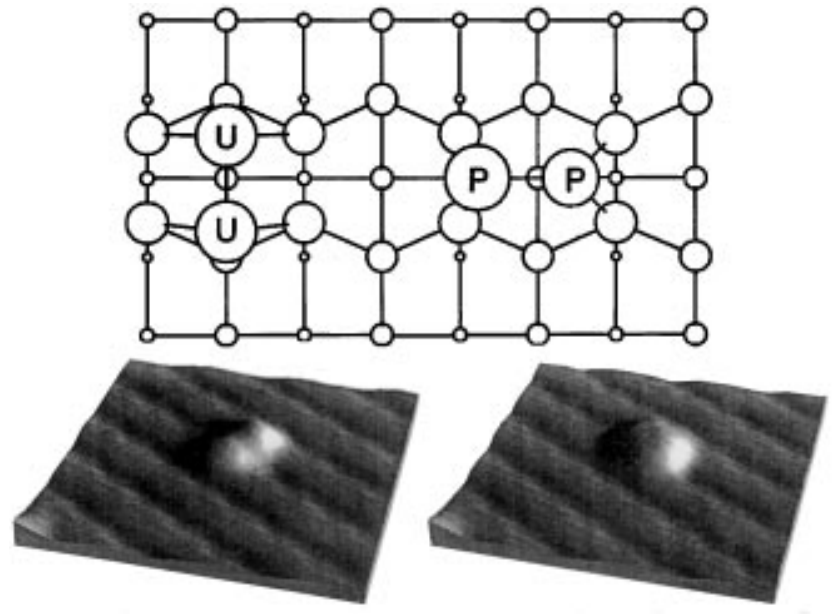

FIG. 1. Top: A schematic of the Si(100) surface showing the $P-P$ and $U-U$ ad-dimer configurations. Circle radii indicate vertical displacement of the atoms. Bottom: Empty state STM images $(50 \AA)$ of an ad-dimer in the $U-U$ (left) and $P$ - $P$ (right) configurations. Sample bias $+2.5 \mathrm{~V}$.

configuration. Bedrossian, Smith, and Jónsson pointed out, based on preliminary calculations, that by going from the LDA to gradient dependent functionals in the DFT calculations the preference for the $U-U$ configuration can be eliminated and reversed depending on which functional is used [14]. Here we present well converged calculations on the energy difference between the two ad-dimer configurations and test the various functionals against the experimental measurements.

To perform quantitative measurements of the dynamics of ad-dimer rotation, we employ a technique called atom tracking in which the STM tip is locked over a selected ad-dimer using lateral feedback [5]. In this mode, the average position of the STM tip is continually maintained over the ad-dimer while the $X, Y$, and $Z$ feedback position data are acquired. Because of the configurational and electronic structure difference between the $U-U$ and $P-P$ orientations the $Z$ feedback position is $0.15 \AA$ closer to the surface over the $U-U$ orientation than the $P-P$ orientation [15]. The state of the ad-dimer is then simply reflected in the $Z$ feedback position as a function of time (Fig. 2). The fact that the tip remains constantly over the ad-dimer during the measurements serves two purposes: first, the rate at which dynamic events (in this

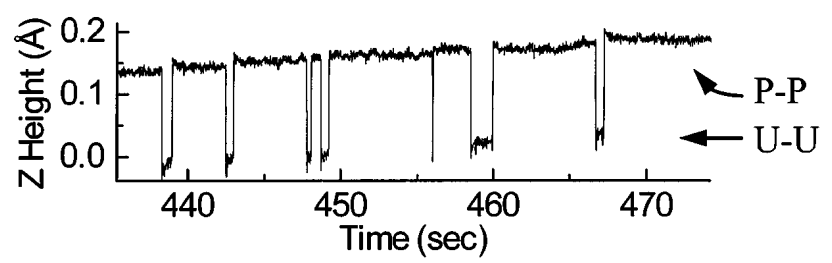

FIG. 2. The measured height of the tip above the surface as a function of time, showing the transitions between the $P-P$ and $U-U$ states. case ad-dimer rotation) can be resolved is increased by about a factor of 1000 over conventional STM imaging techniques, and, second, it allows the dependence of the kinetics on the applied tunneling parameters to be easily measured. The STM is operated in constant current mode so that the magnitude of the electric field can be adjusted by changing the demanded current and/or the bias voltage. The relative change in the tip-sample separation as a function of current and bias can be measured accurately; however, the absolute tip-sample separation is ill defined. In this Letter we assume an initial tip-sample separation of $5 \AA$ for a sample bias of $2.5 \mathrm{~V}$ and $0.04 \mathrm{nA}$ tunneling current giving an electric field of $0.5 \mathrm{~V} / \AA$. The change in the field due to changing the tunneling parameters is calculated using the measured change in the tip-sample separation from this initial offset.

The theoretical calculations are performed with a slab geometry subject to periodic boundary conditions and a plane wave basis set. For most of our calculations, the unit cell consists of eight layers of eight atoms each, with the lowest three layers frozen and with a $10 \AA$ vacuum spacing between slabs. The electronic and ionic degrees of freedom are relaxed simultaneously using the Car-Parrinello approach [16] and also with an all bands iterative scheme based on the Davidson method. Norm conserving pseudopotentials [17] with $s$ and $p$ nonlocality are employed to model the core (i.e., $1 s, 2 s$, and $2 p$ ) electrons of $\mathrm{Si}$, and the basis set cutoff is $12 \mathrm{Ry}$. Up to four $k$ points are included in the plane wave representation of the wave function. We also performed calculations with a much larger simulation cell (including 32 atoms per layer), a slightly different pseudopotential [18], and a smaller, 10 Ry cutoff. We estimate the error in our best converged results is less than $0.05 \mathrm{eV}$ due to these effects. The geometry relaxations are done in the LDA, using the Perdew-Zunger parametrization of the Ceperley-Alder exchange correlation functional [19]. The relaxation calculations are continued until the forces on movable atoms drop to below $10^{-4}$ atomic units, at which point we estimate the energy is converged to $10^{-2} \mathrm{eV}$. Energies are calculated using several gradient dependent functionals [20-22], but the geometries and charge densities are determined by selfconsistent LDA calculations. Our parallelized simulation code is described elsewhere [23].

In Fig. 3(a) we show an Arrhenius plot for a single ad-dimer tracked between room temperature and $63^{\circ} \mathrm{C}$ using a tunneling sample bias of $2.5 \mathrm{~V}$ at $0.04 \mathrm{nA}$. The barrier to rotate out of each stable state is given by the slope of the measured flipping rate versus $1 / k T$. Over this temperature range the flipping rate increases by an order of magnitude. The measured rotational barrier at these tunneling conditions for the $P-P(U-U)$ configuration is $0.70 \pm 0.08 \mathrm{eV}(0.64 \pm 0.09 \mathrm{eV})$ with a frequency prefactor of $10^{10.8 \pm 1.4} \mathrm{sec}^{-1}\left(10^{10.7 \pm 1.3} \mathrm{sec}^{-1}\right)$.

At room temperature we measured the flipping rates as a function of the estimated applied electric field between 

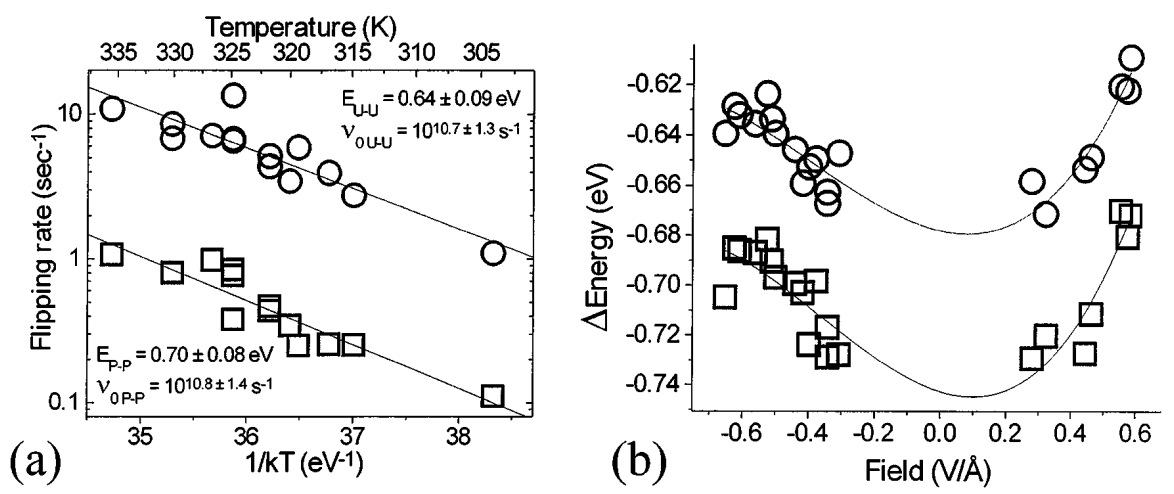

FIG. 3. (a) Arrhenius plot for data taken between room temperature and $65^{\circ} \mathrm{C}$ for a single dimer. (b) The dependence of the activation energy on applied field and a cubic polynomial fit. The circles (squares) designate rotation out of the $U-U(P-P)$ state.

the tip and sample. In Fig. 3(b) we plot the difference in energy between the transition state and the stable states as a function of field for which we used the measured average prefactor of $10^{10.75}$. Three qualitative aspects of this plot are immediately apparent. First, the barrier decreases (flipping rate increases) with an increase in the magnitude of the field regardless of sign. This indicates that the polarizability of the states has a larger effect than the charge of the states. Second, there is an asymmetry between the positive and negative fields with the barrier decreasing more rapidly for positive values. At positive field the tip is biased negative with respect to the sample. And last, the difference in energy between the two stable states is nearly field independent; i.e., the two curves are virtually parallel. This implies that the field is affecting the $P-P$ and $U-U$ states similarly and/or that the dominant field dependence is due to the transition state. Because of this field independence we can immediately extract a value for the difference in free energy between the $P-P$ and $U-U$ orientations, $0.059 \pm 0.009 \mathrm{eV}$. This value is very close to that arrived at by Zhang et al. [12] and Bedrossian [4] by simply counting the populations of the two states in images acquired at room temperature for a single tunneling condition.

The DFT energy calculations [24] using LDA favor the $U-U$ configuration slightly, by $0.06 \mathrm{eV}$, but the $P-P$ is slightly favored, by $0.07 \mathrm{eV}$, when using the gradient dependent PW91 functional [20]. The gradient dependent BLYP functional [20-22], which differs from the PW91 functional mainly in the correlation energy, favors the $P-P$ much more strongly, by $0.3 \mathrm{eV}$. It is surprising how much the calculated energy difference between these very similar configurations depends on which functional is used. The PW91 results are in excellent agreement with the experimental measurements and the LDA results are not in significant disagreement, but the BLYP functional clearly favors the $P-P$ configuration too strongly. This is unexpected since the BLYP functional has been found to give highly accurate results for several molecular systems [25] and, as a result, has become widely used in molecular chemistry calculations.
The observed relative populations of the two rotational states show a very small dependence on the bias field. Therefore, the field appears to have a similar effect on the two states. However, the transition rate between the two states is sensitive to the applied field as shown in Fig. 3(b). The solid lines in Fig. 3(b) are fits of the field dependence to a polynomial from which we extract the zero-field rotational barriers. The intrinsic rotational barrier for the $P-P(U-U)$ state is measured to be $0.74 \pm 0.01 \mathrm{eV}(0.68 \pm 0.01 \mathrm{eV})$. We remark here that these values, although systematically higher, are within the error bars of the finite field values measured from the Arrhenius plot of Fig. 3(a).

Again, we stress that the numerical values for the field are only an estimate. The magnitude of the field at the ad-dimer depends on the tip-sample separation as well as the depth of field penetration into the semiconductor. However, changing the estimated tip-sample separation by as much as a factor of 2 changes the interpolated zero-field barriers by only $10 \mathrm{meV}$. The details of the field and the field gradient also depend upon the unknown details of the tip structure. The data displayed in Fig. 3(b) were acquired with several different tip geometries, and certainly some of the scatter in this data set is due to these differences.

The transition path for the rotation has recently been analyzed theoretically in detail by Brocks and Kelly [11]. From both high temperature dynamics simulations and constrained structural relaxations the optimal path is found to involve direct, asymmetric rotation (not an exchange) and the estimated barrier is $0.9 \mathrm{eV}$ using LDA. At the transition state the height of the ad-dimer is between that of the two stable states, slightly higher than the $U-U$ ad-dimer.

We observe that the polarization effect, second order in the applied field, dominates at the strong fields used in the measurements here. The applied field lowers the activation energy barrier for both positive and negative bias, indicating that the transition state has larger polarizability than the stable configurations. This is to be expected because of a broken bond in the transition state configuration. The 
corresponding, dangling bond electrons are more free to respond to the external field. Because the presence of the electric field alters the charge distribution, it is difficult to make a quantitative comparison between the field dependence of the measured energetics and the current level of calculations presented in this Letter which are performed at zero field. In fact, calculations of the first order effect, proportional to the dipole moment of the ad-dimer evaluated from the DFT electron density, find a field dependence that is an order of magnitude smaller than the observations. Results of field dependent calculations of the electronic and structural response of the stable and transition states in a uniform applied will be presented elsewhere [26].

In summary, we made detailed experimental measurements of the rotation kinetics of Si ad-dimers on the $\mathrm{Si}(001)$ surface as a function of temperature to determine the relative stability and the activation barriers. We compared the measured stability of the two orientations of the $\mathrm{Si}$ ad-dimer with calculations using various density functionals, in particular, different gradient dependent functionals. The PW91 functional is found to give very accurate results while the predictions based on the BLYP functional are in significant disagreement with the measurements. By measuring the rotation rates as a function of applied electric field we find that, although the fields are very large, the barriers are altered by less than $10 \%$.

The experimental work performed at Sandia National Laboratories was supported by the U. S. Department of Energy under Contract No. DE-AC04-94AL85000. The theoretical work was supported by the Department of Energy under Award No. DE-FG06-91ER14224 (A.P.S.) and by the National Science Foundation under Award No. CHE-9217294-CARM (H.J. and A.P.S.). H.J. would like to thank CAMP (Center for Atomic scale Materials Physics) and the Physics Department of the Technical University of Denmark for hospitality and support. Computer time was made available on a Paragon supercomputer by the San Diego Supercomputer Center.

[1] R. A. Wolkow, Phys. Rev. Lett. 74, 4448 (1995).

[2] A. Smith et al., J. Chem. Phys. 102, 1044 (1995); A. P. Smith and H. Jónsson, Phys. Rev. Lett. 77, 1326 (1996).
[3] Y.W. Mo et al., Phys. Rev. Lett. 63, 2393 (1989); 66, 1998 (1991).

[4] P. J. Bedrossian, Phys. Rev. Lett. 74, 3648 (1995).

[5] B. S. Swartzentruber, Phys. Rev. Lett. 76, 459 (1996).

[6] Recent experimental results show that the ad-dimer position on top of the substrate dimer row is more stable than between the rows. B. S. Swartzentruber (unpublished).

[7] N. Kitamura et al., Phys. Rev. Lett. 71, 2082 (1993).

[8] B. S. Swartzentruber and M. Schacht, Surf. Sci. 322, 83 (1995).

[9] C. Pearson et al., Phys. Rev. Lett. 74, 2710 (1995).

[10] G. Brocks, P. J. Kelly, and R. Car, Surf. Sci. 269/270, 860 (1992).

[11] More recent calculations find an energy difference of $0.01 \mathrm{eV}$. G. Brocks and P. J. Kelly, Phys. Rev. Lett. 76, 2362 (1996).

[12] Z. Zhang et al., Phys. Rev. Lett. 74, 3644 (1995).

[13] F. H. Stillinger and T. A. Weber, Phys. Rev. B 31, 5262 (1985).

[14] P. J. Bedrossian, A. P. Smith, and H. Jónsson, Mater. Res. Soc. Symp. Proc. 389, 35 (1995).

[15] The DFT calculations predict that the upper atom of the highly tilted $P$ - $P$ ad-dimer is about $0.6 \AA$ higher above the surface than the atoms in the $U-U$ ad-dimer.

[16] R. Car and M. Parrinello, Phys. Rev. Lett. 55, 2471 (1985).

[17] G. B. Bachelet, D. R. Hamann, and M. Schluter, Phys. Rev. B 26, 4199 (1982).

[18] N. Troullier and J. L. Martins, Phys. Rev. B 43, 1993 (1991).

[19] J. P. Perdew and A. Zunger, Phys. Rev. B 23, 5048 (1981).

[20] J. P. Perdew, in Electronic Structure of Solids '91, edited by P. Ziesche and H. Eschrig (Akademie Verlag, Berlin, 1991); J. P. Perdew and Y. Wang (unpublished).

[21] A. D. Becke, Phys. Rev. A 38, 3098 (1988).

[22] C. Lee, W. Yang, and R. G. Parr, Phys. Rev. B 37, 785 (1988).

[23] J. K. Wiggs and H. Jónsson, Comput. Phys. Commun. 81, 1 (1994); 87, 319 (1995).

[24] We neglect here entropic contributions to the relative stability; those are expected to be on the order of $k T=$ $0.03 \mathrm{eV}$ or smaller.

[25] B. G. Johnson, P. M. W. Gill, and J. A. Pople, J. Chem. Phys. 98, 512 (1993); K. Kim and K. D. Jordan, J. Phys. Chem. 98, 10089 (1994); P. Nachtigall, K. D. Jordan, A. P. Smith, and H. Jónsson, J. Chem. Phys. 104, 148 (1996).

[26] H. Jonsson and J. K. Norskov (unpublished). 